\title{
Hirudin Suppresses Metastasis of Human Breast Adenocarcinoma Cells In Vitro and in a Zebrafish Xenograft Model
}

\section{Shuo Zhang}

Nantong Hospital of Traditional Chinese Medicine

Lei Zhou

Nantong Hospital of Traditional Chinese Medicine

XiaoYan Jiang ( 992113053@qq.com )

Nantong Hospital of Traditional Chinese Medicine

HongHui Ni

Nantong Hospital of Traditional Chinese Medicine

ShuiYing Yang

Nantong Hospital of Traditional Chinese Medicine

Yan Xu

Nantong Hospital of Traditional Chinese Medicine

Yu Wu

Nantong Hospital of Traditional Chinese Medicine

Dan Du

Nantong Hospital of Traditional Chinese Medicine

Min Peng

Nantong Hospital of Traditional Chinese Medicine

YingJun Fu

Nantong Hospital of Traditional Chinese Medicine

Yan Cai

Nantong Hospital of Traditional Chinese Medicine

XiaoHui Yang

Nantong Hospital of Traditional Chinese Medicine

\section{Research Article}

Keywords: Breast cancer, Hirudin, Metastasis , Zebrafish , CHD1L

Posted Date: May 13th, 2021

DOI: https://doi.org/10.21203/rs.3.rs-441376/v1 
License: (c) (i) This work is licensed under a Creative Commons Attribution 4.0 International License. Read Full License 
1 Hirudin Suppresses Metastasis of Human Breast Adenocarcinoma

2 Cells In Vitro and in a Zebrafish Xenograft Model

3

4 Shuo Zhang", Lei Zhou\#, XiaoYan Jiang*, HongHui Ni, ShuiYing Yang, Yan Xu, Yu

5 Wu, Dan Du, Min Peng, YingJun Fu, Yan Cai, XiaoHui Yang

6

7

Pharmacy Department, Nantong Hospital of Traditional Chinese Medicine, Nantong, Jiangsu, P.R. China.

${ }^{\#}$ The two authors contributed equally to this work.

*Correspondence: $992113053 @ q q . c o m$

Pharmacy department, Nantong Hospital of Traditional Chinese Medicine, 41 Jianshe Road, Nantong, Jiangsu 226001, P.R. China

\section{Abstract}

Background: Breast cancer is the leading cause of cancer-related death in women worldwide. Hirudin has been shown to inhibit the growth and metastasis of several types of cancers in experimental tumor models. However, whether hirudin exerts antitumor effects on breast adenocarcinoma cells has not yet been investigated. The objective of this study was to evaluate the antitumor effects and explore the underlying mechanisms of hirudin in breast adenocarcinoma MCF-7 cells.

Methods: The viability of MCF-7 cells was assayed by Cell Counting Kit-8. The adhesion ability of the cells was evaluated by cell adhesion assay. Besides, cell migration was detected by wound healing assay. Cell invasion was examined using Transwell chamber assay. The underlying molecular mechanism was investigated by immunofluorescence. In addition, In vivo zebrafish xenograft model was used to verify the proliferation and metastasis of hirudin on MCF-7 cells.

Results: The results showed that hirudin significantly inhibited the cell viability and suppressed cell adhesion, migration, invasion compared with the control group. Importantly, hirudin significantly decreased the expression of CHD1L, MDM2 protein, and increased the expression of p53 protein. Moreover, the zebrafish xenograft study revealed that hirudin inhibited the proliferation and metastasis of MCF-7 cells in vivo. 
Conclusion: The present findings demonstrate that hirudin suppressed metastasis of MCF-7 cells and the mechanism may involve with the CHD1L/MDM2/p53 axis. Hirudin is a promising antineoplastic agent for the treatment of breast cancer with significant antimetastatic activities.

Keywords: Breast cancer, Hirudin, Metastasis, Zebrafish, CHD1L

\section{Introduction}

Breast cancer was the most commonly diagnosed cancer $(24.2 \%$ of the total cancer cases) and the leading cause of cancer-related death (15\% of the total cancer deaths) among females worldwide in $2018^{[1]}$. Metastatic disease accounts for more than $90 \%$ of breast cancer-related deaths. It has been reported that the occurrence of breast cancer is closely related to the instability of the genome, such as gene amplification, deletion, mutation ${ }^{[2]}$. Therefore, it is of great significance to further study and reveal the key targets and regulatory mechanisms of tumor metastasis for seeking more effective treatment.

Chromodomain helicase/ATP DNA binding protein 1-like gene (CHD1L) is a member of the SNF-2 protein family, which exhibits an oncogenic role during malignant transformation ${ }^{[3]}$. CHD1L is overexpressed in many highly aggressive tumors, such as breast cancer, colorectal cancer and intrahepatic cholangiocarcinoma [4-6]. Therefore, CHD1L is a novel biomarker for predicting tumor progression, prognosis, and survival. Murine double minute 2 (MDM2), an important downstream target of CHD1L, is an oncogene closely related to malignant tumors. It is a ubiquitin-protein ligase that is involved in the identification of target proteins by ubiquitin, leading to the degradation of target proteins through the proteasome. The protein encoded by MDM2 can bind to p53 protein and negatively regulate p53 protein. It leads to the inactivation of tumor suppressor gene p53, and ultimately enhances the ability of cell transformation, proliferation and malignant transformation. The first MDM2 inhibitor to enter human clinical trials, RG7112, can achieve good pharmacokinetic properties by oral administration ${ }^{[7]}$. Meanwhile, the activation of $\mathrm{p} 53$, the increase of $\mathrm{p} 21$ protein and the induction of apoptosis were detected in tumor cells, which effectively inhibited the growth of cancer cell lines ${ }^{[8]}$. In conclusion, CHD1L promotes the progression of tumor cells through the MDM2/p53 signaling pathway.

Hirudin is the main active component of animal medicine hirudo, and has demonstrated antitumor effects in different types of tumors including lung, laryngeal 
cancer $^{[9-10]}$. Thus, targeting hirudin is a promising strategy for the development of anticancer therapeutic modalities. However, whether hirudin exerts antitumor effects on breast adenocarcinoma cells has not yet been investigated. In the present study, we evaluated the antitumor effects of hirudin and explored the underlying mechanisms in breast adenocarcinoma cells. We treated MCF-7 cells with various dosages of hirudin and analyzed cell viability, adhesion, migration and invasion. We additionally assessed the proliferation and metastasis of hirudin on MCF-7 cells in vivo zebrafish xenograft model.

\section{Materials and Methods}

\section{Cell culture and reagents}

The human breast adenocarcinoma MCF-7 cells were provided by China Infrastructure of Cell Line Resource. Cells were cultured in RPMI-1640 medium (Gibco, USA) with 10\% FBS (Gibco) plus $1 \%$ penicillin and streptomycin in a $5 \%$ $\mathrm{CO}_{2}$ atmosphere at $37^{\circ} \mathrm{C}$. Hirudin (purity $\geqslant 95 \%$ ) was purchased from Combination Botai Biotechnology (Dalian, China).

\section{Cell counting Kit-8 assay}

The viability of breast adenocarcinoma cells was assayed by Cell Counting Kit-8 (CCK-8; Dojindo, Beijing, China) assay. First, $5 \times 10^{3}$ cells/well were added to a 96-well plate, with $100 \mu 1$ of suspension in each well. Twenty-four hours after seeding, the medium was replaced with media containing different concentrations of hirudin created through serial dilution. After culturing the cells for $24 \mathrm{~h}, 48 \mathrm{~h}$, and $72 \mathrm{~h}, 10 \mu \mathrm{l}$ of CCK-8 solution was added to each well, and the cells were incubated for $30 \mathrm{~min}$. Optical density (OD) was measured at $450 \mathrm{~nm}$ with a microplate reader, and the inhibition rate was calculated as follows: Relative cell viability inhibition rate $(\%)=$ Absorbance of experimental group/ Absorbance of control group) $\times 100 \%$.

\section{Cell adhesion assay}

MCF7 cells were plated in 6-well culture dishes with or without hirudin and allowed to adhere for $1 \mathrm{~h}$. Subsequently, corresponding medium with non-adhered cells was discarded and cells were gently washed twice with PBS in order to remove any loosely attached cells. Adhered cells were then counted using a $0.1 \%$ crystal violet staining solution at room temperature for $20 \mathrm{~min}$ subsequent to being fixed with methanol for $30 \mathrm{~min}$ at room temperature. Data are expressed as a percentage in adhered cells treated with hirudin relative to the control cells.

\section{Wound healing assay}


Cell migration was analyzed using a wound healing assay. MCF7 cells $\left(1.5 \times 10^{5}\right)$ were seeded onto 12-well plates until they reached confluence. A scratch wound in confluent monolayer was made using a $10 \mu \mathrm{l}$ sterile pipette tip. Subsequent to washing away all detached cells with PBS, the remaining cells were treated with or without hirudin $(0.5,1,2 \mathrm{mg} / \mathrm{ml})$. Photographs were taken at 0 and $24 \mathrm{~h}$ after treatment.

\section{Transwell chamber assay}

Invasion assay was performed using 24-well Transwell chambers with 8 - $\mu \mathrm{m}$ pore filters (Corning, USA). The inserts were coated with a $50 \mathrm{mg} / 1$ Matrigel (1: 4 dilution, BD, USA) and cultured at $37^{\circ} \mathrm{C}$ for $2 \mathrm{~h}$. Cells $\left(1 \times 10^{5} / \mathrm{ml}\right)$ suspended in $200 \mu \mathrm{l}$ of serum-free RPMI1640 medium were transferred to the upper chamber, while $600 \mu 1$ of RPMI1640 medium supplemented with 20\% FBS (fetal bovine serum) was added to the lower chamber as a chemoattractant. The medium was then discarded and invaded cells were fixed with $4 \%$ paraformaldehyde and stained with $0.1 \%$ crystal violet. The invading cells were counted in 5 different randomly selected $200 \times$ magnification fields under light microscopy.

\section{Immunofluorescence}

Cells at a density of $1 \times 10^{4}$ cells/well were seeded into 96-well plates. After exposure to the indicated treatments, the cells were fixed with $4 \%$ paraformaldehyde for $15 \mathrm{~min}$ at room temperature. Then, the cells were blocked with $5 \%$ goat serum and $0.3 \%$ Triton X-100 in PBS for $1 \mathrm{~h}$. After the blocking solution was aspirated, the cells were incubated with a primary antibody against CHD1L (1:500, Abcam, UK), MDM2 (1:400, Abcam, UK) and p53 (1:100, Abcam, UK) overnight at $4^{\circ} \mathrm{C}$. The next day, the cells were incubated with a fluorochrome-conjugated anti-rabbit secondary antibody (1:1000, CST, US) for $2 \mathrm{~h}$ at room temperature in the dark. Subsequently, the cells were stained with DAPI (Beyotime, Shanghai, China) for $30 \mathrm{~min}$. Images were obtained under a fluorescence microscope.

\section{Zebrafish embryos}

Wild type $\mathrm{AB}$ strain of zebrafish (Danio rerio) was obtained from Nanjing Xinjia Biotechnology Co., Nanjing, China. Embryos were generated by natural pairwise mating. Zebrafishes were cultured under photoperiod of $14 \mathrm{~h}$ light $/ 10 \mathrm{~h}$ dark at $28^{\circ} \mathrm{C}$. After disinfection and washing, the fertilized eggs were transferred into zebrafish embryo culture water.

\section{In Vivo Antitumor Assay}


Fluorescence-labeled MCF-7 cells were injected into the abdominal perivitelline space of zebrafish embryos with a nanoliter injector. The tumor-bearing embryos were incubated at $0,0.05,0.5,5 \mathrm{mg} / \mathrm{mL}$ hirudin $24 \mathrm{~h}$ after transplantation. The tumor growth and metastasis were observed and imaged using fluorescence inverted microscope (Nikon Inc., Tokyo, Japan). The mean fluorescence intensity was analyzed using Image $\mathbf{J}$ software.

\section{Statistical analysis}

All data were expressed as means \pm Standard Deviation (SD). Statistical analysis was performed by one-way analysis of variance based on Student's two-tailed unpaired t-test or Dunnett's multiple comparisons test using GraphPad Prism 5.0 software (GraphPad Software, CA, USA). Image-Pro Plus software 6.0 (Media Cybernetics, Inc., Silver Spring, MD, USA) was used for processing images. A value of $P<0.05$ was considered to be a statistically significant difference for all tests.

\section{Results}

\section{Hirudin inhibits the proliferation of MCF7 cells}

The effect of hirudin on cell growth in human MCF7 cells was detected by CCK-8 assay. After treatment with hirudin varying from 0.25 to $2 \mathrm{mg} / \mathrm{mL}$ after 24,48 , and $72 \mathrm{~h}$, the results revealed that cell viability was inhibited by hirudin compared with the control group $(p<0.05)$ (Figure 1).

\section{Hirudin suppresses the adhesion, migration and invasion of MCF7 cells}

In the present study, a non-specific cell adhesion assay was used to investigate the effect of hirudin on cell attachment. Compared with the control group, hirudin significantly inhibited the adhesion of MCF7 cells $(p<0.01)$ (Figure 2). Wound healing assay was performed to determine the effects of hirudin on migration of MCF7 cells. Migration of MCF7 cells treated with hirudin was blocked compared with the control group $(p<0.01)$ (Figure 3$)$. In a consistent manner, hirudin suppressed invasion of MCF7 cells $(p<0.01)$ (Figure 4). Taken together, these results demonstrated that hirudin by acting directly on MCF7 cells to inhibit the processes of adhesion, migration and invasion.

\section{Hirudin suppressed the protein levels of CHD1L and MDM2, upregulated the} protein levels of p53

The expression of protein levels was detected by immunofluorescence. As shown in Figure 5, hirudin significantly decreased the expression of CHD1L, MDM2 protein, 
and increased the expression of $\mathrm{p} 53$ protein $(p<0.05$ or $p<0.01)$.

\section{Hirudin inhibited tumor growth and metastasis in the zebrafish xenograft model} In vivo zebrafish xenograft model was used to verify the proliferation and metastasis of hirudin on MCF-7 cells. Fluorescence-labeled MCF-7 cells were localized by fluorescence imaging and expression intensities were processed with image analysis software. Compared with the control group, hirudin $(0.5-5 \mathrm{mg} / \mathrm{mL})$ significantly inhibited the proliferation of MCF-7 cells, while $0.05 \mathrm{mg} / \mathrm{mL}$ did not (Figures 6A and 6B). Meanwhile, decreasing metastatic incidences in tumor-bearing zebrafishes were detected with rising concentration of hirudin (Figures 6C and 6D).

\section{Discussion}

Breast cancer usually has a poor clinical outcome and low survival rate due to high rates of metastasis at a late stage of the tumor development. Therefore, novel approaches are urgently required for the treatment of this disease and the prevention of its metastasis ${ }^{[11]}$. Animal drugs have widely attracted attention due to their preventative and treatment effects on cancer without serious side effects in recent years $^{[12]}$. The results of the present study demonstrated that animal medicine hirudin served an important functional role in suppressing the invasion and metastasis of breast adenocarcinoma cells.

Cancer metastasis is a complicated multistep process involving the dissociation of malignant cells at the primary sites, invasion through the extracellular matrix, intravasation of invading cells into the vasculature or lymphatic systems, survival and finally proliferation at a distant organ ${ }^{[13]}$. In the present study, it was revealed that the cell proliferation, adhesion, migration, and invasion in MCF-7 cells decreased when treated with hirudin.

We explored the possible explanation for its suppression of human breast adenocarcinoma MCF-7 cells migration and invasion. Recent studies have shown that CHD1L is related to proliferation, migration, invasion, and metastasis of tumor cells ${ }^{[14-15]}$. Therefore CHD1L may become a new independent marker of tumor progression, prognosis, and survival time. The role of the CHD1L/MDM2/p53 pathway loop in cancers has been widely studied. p53 coordinates a transcription program to stall the cell cycle, promote DNA repair, and initiate senescence or apoptosis. The primary modulators of p53 activity is the E3 ubiquitin-protein ligase MDM2 which constitutively polyubiquitinate p53 for proteasomal degradation, 
maintaining p53 at low levels ${ }^{[16]}$. Our result suggests that down-regulation of CHD1L, MDM2 and up-regulation of p53 might be responsible for the anti-metastasis mechanisms of hirudin in breast cancer.

The zebrafish has become a powerful vertebrate system for modeling human cancers and is an excellent platform to study all stages of metastasis ${ }^{[17]}$. The transplantation of cancer cells into zebrafish embryos is most frequently used in zebrafish models. Briefly, human cancer cells labeled with a fluorescent marker are inoculated into larvae or embryos of zebrafish ${ }^{[18]}$. Hence, the purpose of the study was to confirm anti-proliferation and anti-metastasis effect of hirudin using a zebrafish xenograft model.

In conclusion, the present study demonstrated that hirudin may inhibit the viability, migration and invasion of breast adenocarcinoma cells in vitro and in vivo. Furthermore, it was revealed that the inhibitory effect of hirudin was primarily associated with the suppression of CHD1L, MDM2 and an increase in p53. These results suggested that hirudin may possess great potential for the treatment of breast cancer metastasis partially through CHD1L/MDM2/p53 signaling.

\section{Abbreviations}

CHD1L: Chromodomain helicase/ATP DNA binding protein 1-like; MDM2: Murine double minute 2; FBS: Fetal bovine serum.

\section{Ethics approval}

The study was carried out in compliance with the ARRIVE guidelines. All methods were performed in accordance with the Declaration of Helsinki and Use Committee of the Zebrafish Model Animal Facility, Nanjing University of Chinese Medicine.

\section{Consent for publication}

Not applicable.

\section{Availability of data and materials}

All data of this study is included in this article.

\section{Competing interests}

The authors declare that they have no competing interests. 


\section{Funding}

This study was supported by the Nantong Health Committee (no. QA2019024, JC2019135).

\section{Authors' contributions}

Conceived and designed the study: SZ, XYJ, HHN, SYY. Performed the study: SZ, LZ, YX, YW. Analyzed the data: DD, MP, YJF. Wrote the paper: SZ, YC, XHY. All authors read and approved the final manuscript.

\section{Acknowledgements}

Not Applicable.

\section{Authors' information}

Shuo Zhang, Email: zhangshuo90516@126.com

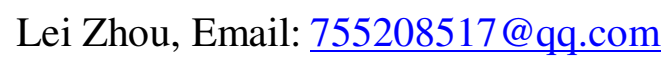

XiaoYan Jiang, Email: $992113053 @ q q . c o m$

HongHui Ni, Email: 2399356123@qq.com

ShuiYing Yang, Email: 2438756110@qq.com

Yan Xu, Email: $1229490753 @ q q . c o m$

Yu Wu, Email: 593048928@qq.com

Dan Du, Email: 7562959@qq.com

Min Peng, Email: 642676355@qq.com

YingJun Fu, Email: $\underline{624894225 @ q q . c o m}$

Yan Cai, Email: 240363544@qq.com

XiaoHui Yang, Email: $594832001 @$ qq.com

\section{References}

[1] $\mathrm{Xu} \mathrm{X}$, Zhang M, Xu F, et al. Wnt signaling in breast cancer: biological mechanisms, challenges and opportunities[J]. Mol Cancer, 2020, doi: 10.1186/s12943-020-01276-5.

[2] Goncalves R, Warner WA, Luo J, et al. New concepts in breast cancer genomics and genetics[J]. Breast Cancer Res, 2014, doi: 10.1186/s13058-014-0460-4.

[3] Zhang L, Jiang Y, Jiao P, et al. The high expression of CHD1L and its clinical significance in human solid tumors: A meta-analysis [J]. Medicine (Baltimore), 
2021, doi: 10.1097/MD.0000000000024851.

[4] Wang W, Wu J, Fei X, et al. CHD1L promotes cell cycle progression and cell motility by up-regulating MDM2 in breast cancer [J]. Am J Transl Res, 2019, 11(3): 1581-1592.

[5] Abbott JM, Zhou Q, Esquer H, et al. First-in-Class Inhibitors of Oncogenic CHD1L With Preclinical Activity Against Colorectal Cancer [J]. Mol Cancer Ther, 2020, 19(8): 1598-1612.

[6] Li S, Chai Y, Ding Y, et al. CHD1L is associated with poor survival and promotes the proliferation and metastasis of intrahepatic cholangiocarcinoma [J]. Oncol Rep, 2020, 42(2): 657-669.

[7] Al-Ghabkari A, Narendran A. In Vitro Characterization of a Potent p53-MDM2 Inhibitor, RG7112 in Neuroblastoma Cancer Cell Lines [J]. Cancer Biother Radiopharm, 2019, 34(4): 252-257.

[8] Verreault M, Schmitt C, Goldwirt L, et al. Preclinical Efficacy of the MDM2 Inhibitor RG7112 in MDM2-Amplified and TP53 Wild-type Glioblastomas [J]. Clin Cancer Res, 2016, 22(5): 1185-1196.

[9] Zhao B, Wu M, Hu Z, et al. Thrombin is a therapeutic target for non-small-cell lung cancer to inhibit vasculogenic mimicry formation [J]. Signal Transduct Target Ther, 2020, doi: 10.1038/s41392-020-0167-1.

[10] Lu Q, Lv M, Xu E, et al. Recombinant hirudin suppresses the viability, adhesion, migration and invasion of Hep-2 human laryngeal cancer cells [J]. Oncol Rep, 2015, 33(3): 1358-1364.

[11] Wang N, Liu W, Zheng Y, et al. CXCL1 derived from tumor-associated macrophages promotes breast cancer metastasis via activating NF- $\mathrm{B} / \mathrm{SOX} 4$ signaling [J]. Cell Death Dis, 2018, doi: 10.1038/s41419-018-0876-3.

[12] Ma Y, Sun X, Yang Y, et al. Prof. Wang Xiaomin's experiences in the treatment of cancer with insect drugs [J]. WJIM, 2017, 12(10): 1378-1380.

[13] Zhou Q, Zhang Z, Song L, et al. Cordyceps militaris fraction inhibits the invasion and metastasis of lung cancer cells through the protein kinase B/glycogen synthase kinase 3 $\beta / \beta$-catenin signaling pathway [J]. Oncol Lett, 2018, 16(6): 6930-6939.

[14] $\mathrm{Xu} \mathrm{X,} \mathrm{He} \mathrm{Y,} \mathrm{Miao} \mathrm{X,} \mathrm{et} \mathrm{al.} \mathrm{Cell} \mathrm{adhesion} \mathrm{induces} \mathrm{overexpression} \mathrm{of}$ chromodomain helicase/ATPase DNA binding protein 1-like gene (CHD1L) and contributes to cell adhesion-mediated drug resistance (CAM-DR) in multiple 
myeloma cells [J]. Leuk Res, 2016, 47: 54-62.

[15] Liu M, Chen L, Ma NF, et al. CHD1L promotes lineage reversion of hepatocellular carcinoma through opening chromatin for key developmental transcription factors [J]. Hepatology, 2016, 63: 1544-1559.

[16] Bauer NC, Anli Yang, Wang X, et al. A cross-nearest neighbor/Monte Carlo algorithm for single-molecule localization microscopy defines interactions between p53, Mdm2, and MEG3 [J]. J Biol Chem, 2021, doi: 10.1016/j.jbc.2021.100540.

[17] Astell KR, Sieger D. Zebrafish In Vivo Models of Cancer and Metastasis [J]. Cold Spring Harb Perspect Med, 2020, doi: 10.1101/cshperspect.a037077.

[18] Nakayama J, Makinoshima H. Zebrafish-Based Screening Models for the Identification of Anti-Metastatic Drugs [J]. Molecules, 2020, doi: 10.3390/molecules25102407.

\section{Figure legends}

Figure 1. Hirudin induces growth inhibition of human breast carcinoma cell line MCF-7 following exposure at different concentrations at 24h, 48h, and $72 \mathrm{~h}$. Results are presented as the mean $\pm \mathrm{SD}$.

Figure 2. Hirudin inhibits the adhesive ability of MCF-7 cells. Data are expressed as a percentage in adhered cells treated with hirudin relative to the control cells. Results are presented as the mean $\pm \mathrm{SD}$. $* * p<0.01$ vs. control group.

Figure 3. Hirudin inhibits the migration ability of MCF-7 cells. (A) Representative images of wound healing at 0 and $24 \mathrm{~h}$. (B) The percentage of migrated cells were calculated based on the number of untreated cells, which were set as $100 \%$. ${ }^{* *} p<0.01$ vs. control group.

Figure 4. Hirudin inhibits the invasion ability of MCF-7 cells. Representative images (A) and data statistics of cells that invaded through the Matrigel coated Transwell chamber (B). ${ }^{* *} p<0.01$ vs. control group.

Figure 5. Hirudin suppressed the protein levels of CHD1L and MDM2, upregulated the protein levels of p53. Representative images (A, C, E) and data statistics of average optical density value $(\mathrm{B}, \mathrm{D}, \mathrm{F}) .{ }^{*} p<0.05$ vs. control group; ${ }^{* *} p<0.01$ vs. control group. 
Figure 6. Evaluation of the in vivo antitumor effects of hirudin in zebrafish xenografted with the human breast carcinoma cell line MCF-7. (A) The in vivo growth is indicated by yellow stained tumor cells detected with the fluorescence microscope. (B) Area of MCF-7 proliferation. Results are presented as the mean \pm SD. ${ }^{* *} p<0.01$ vs. control group; ${ }^{\# \#} p<0.01$ vs. $0.05 \mathrm{mg} / \mathrm{mL}$ group. (C) The in vivo metastasis is indicated by yellow stained tumor cells detected with the fluorescence microscope. (D) Area of MCF-7 metastasis. Results are presented as the mean \pm SD. ${ }^{* *} p<0.01$ vs. control group; ${ }^{\#} p<0.05$ vs. $0.05 \mathrm{mg} / \mathrm{mL}$ group; ${ }^{\#} p<0.01$ vs. $0.05 \mathrm{mg} / \mathrm{mL}$ group.

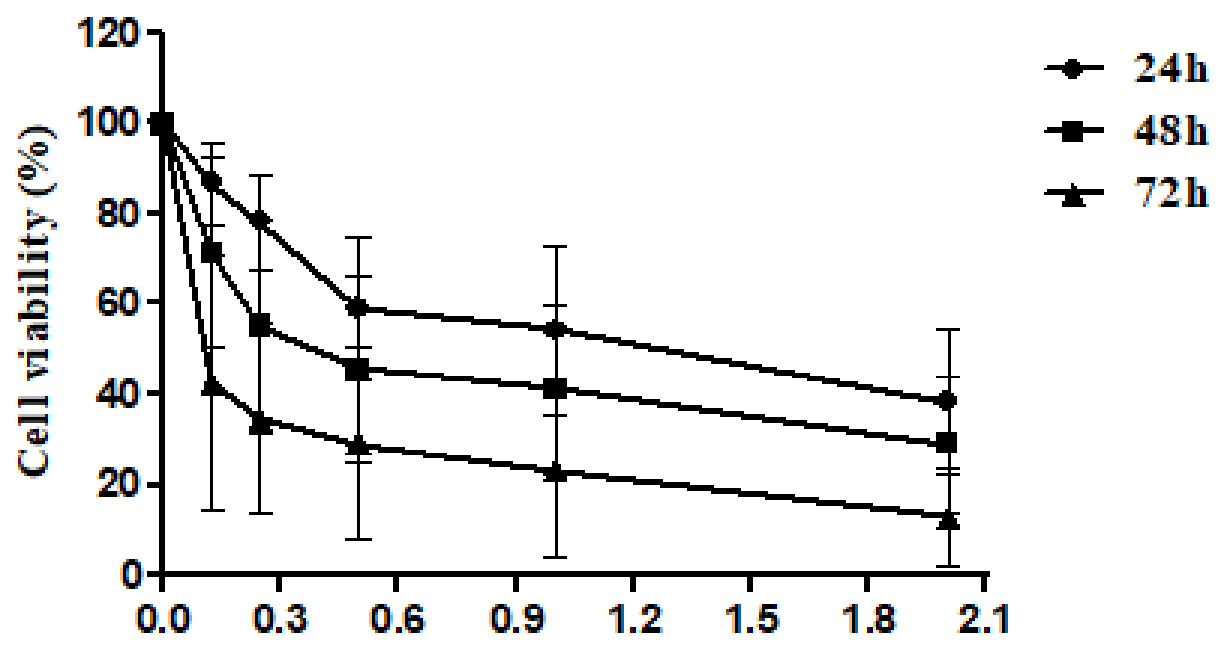

Hirudin concentration $(\mathrm{mg} / \mathrm{mL})$ 
Figure 2.

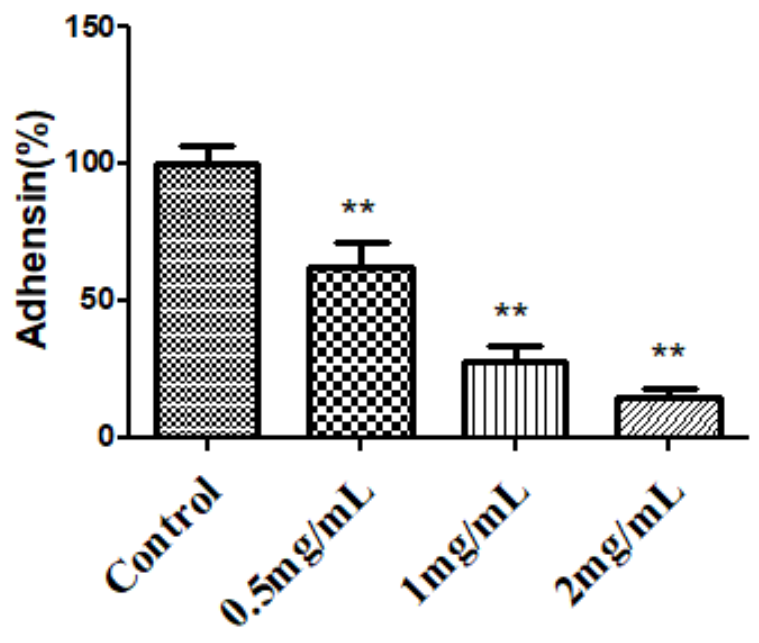


Figure 3.

A
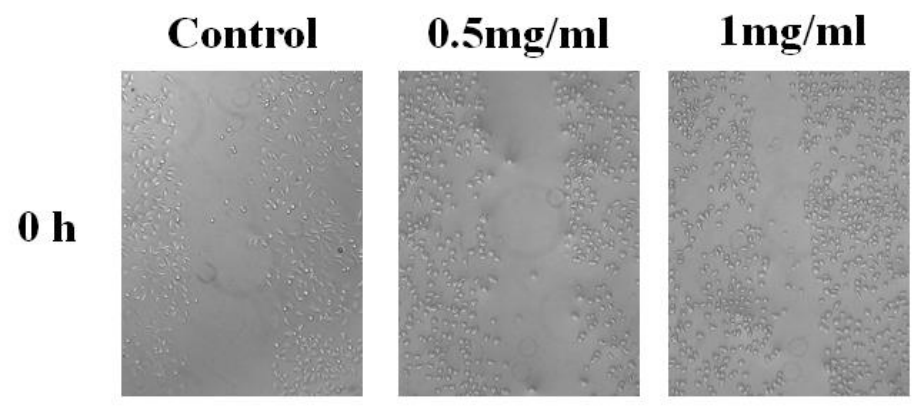

$2 \mathrm{mg} / \mathrm{ml}$
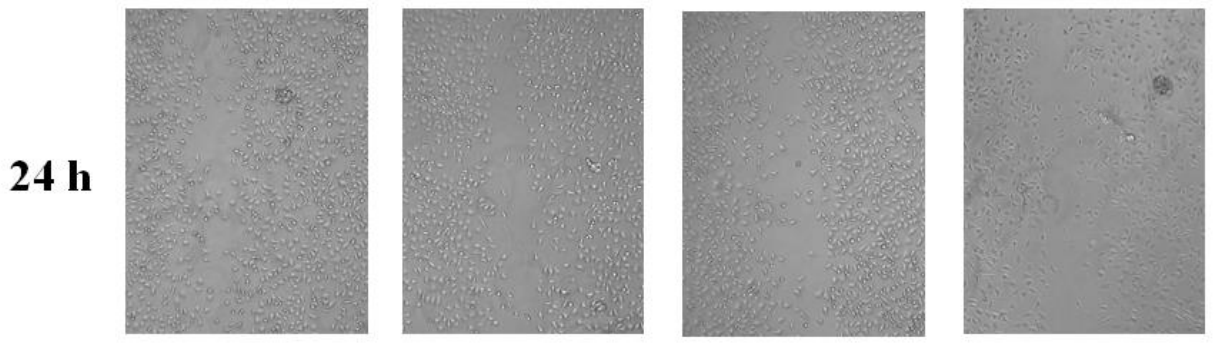

B

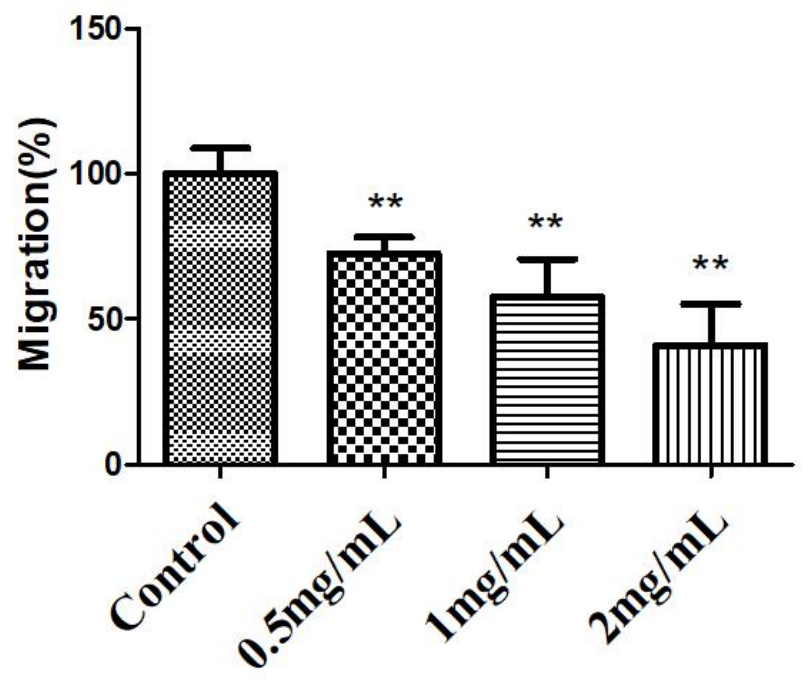

360 
Figure 4.

363
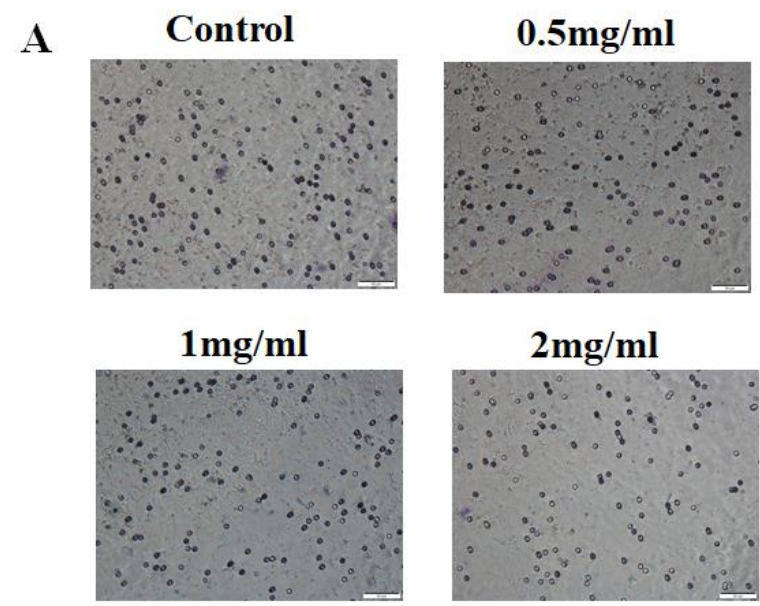

B

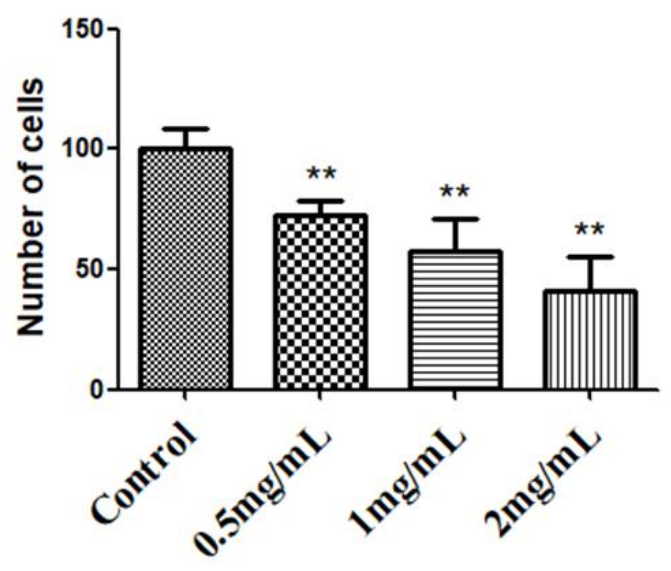

364 
$365 \quad$ Figure 5.

A
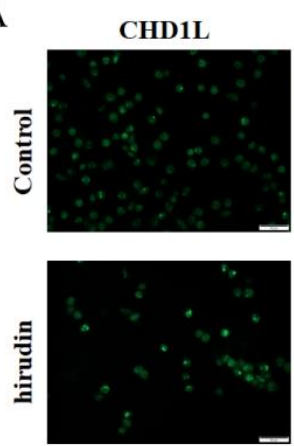

C
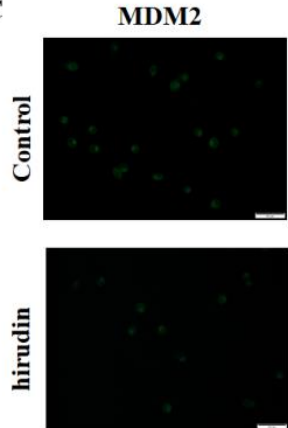

E
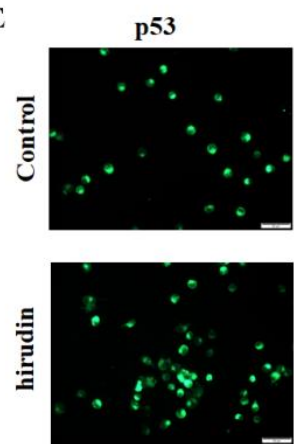

DAPI
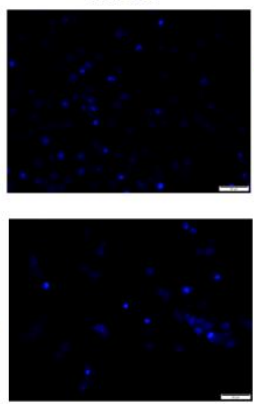

DAPI
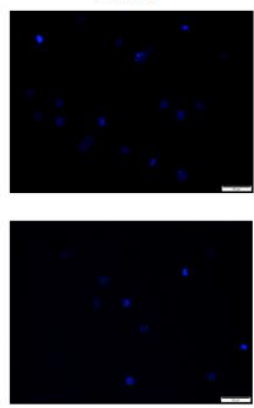

DAPI
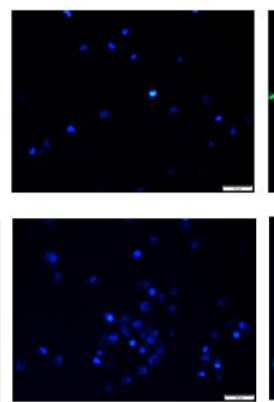

Merge
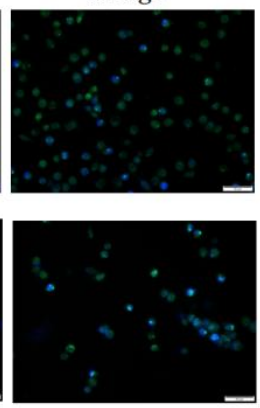

Merge
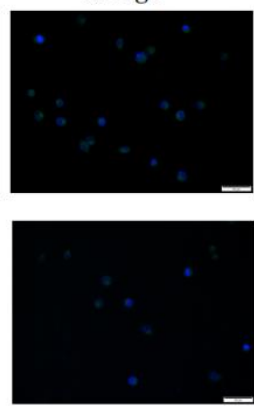

Merge
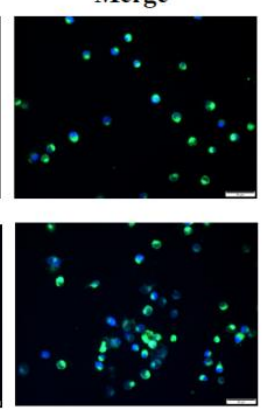

B

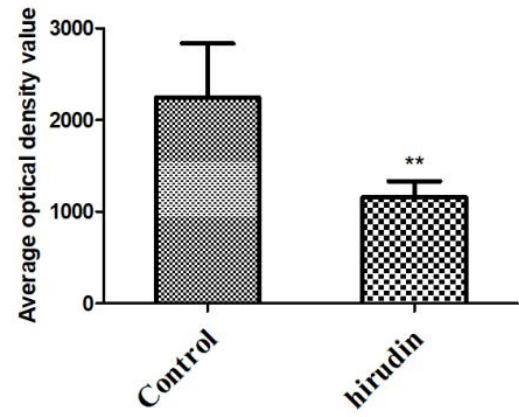

D

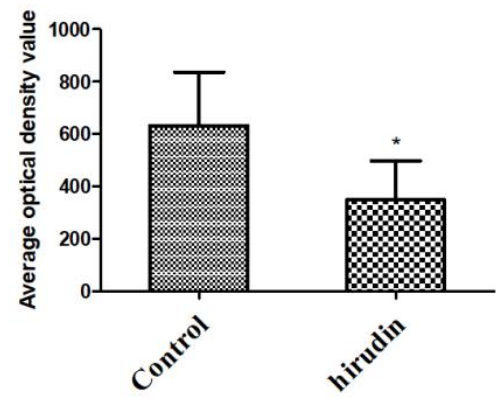

F

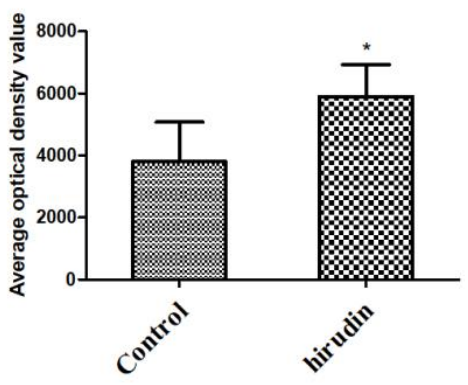


Figure 6.

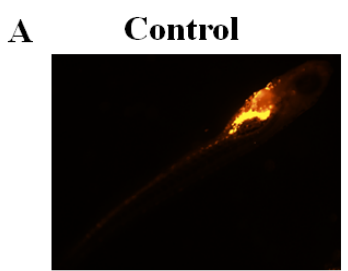

$0.5 \mathrm{mg} / \mathrm{mL}$

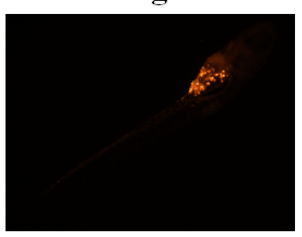

B
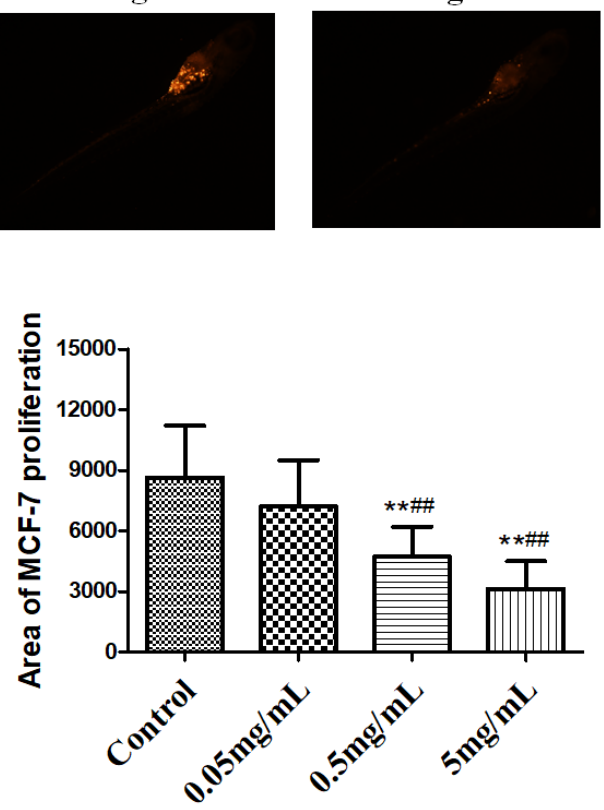
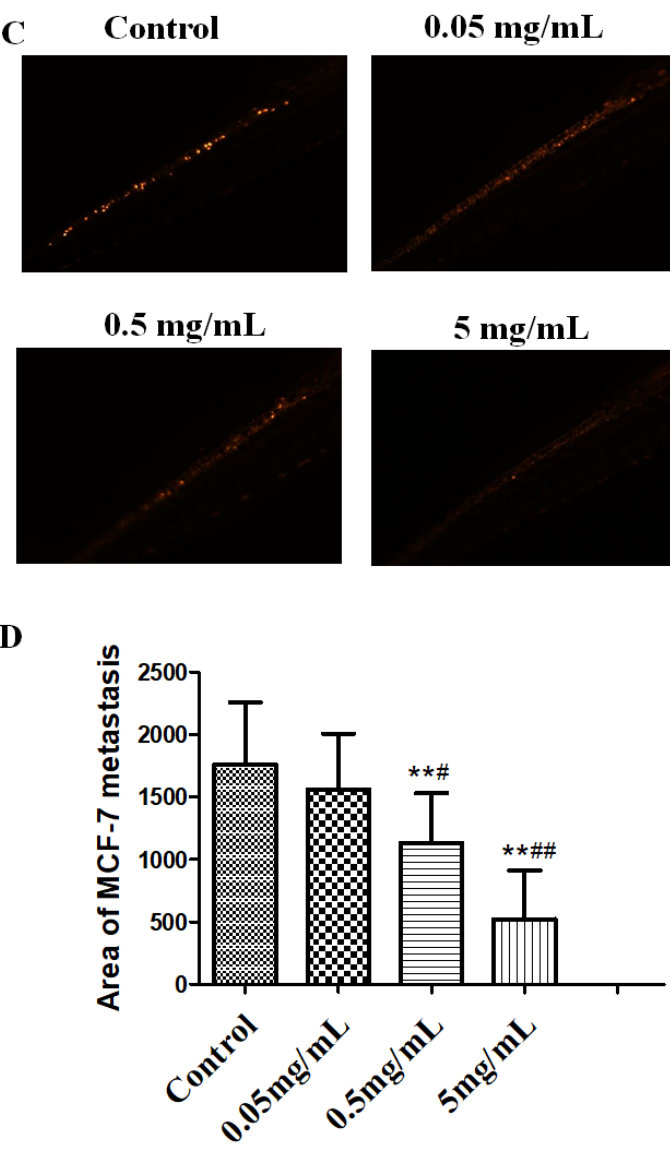

D

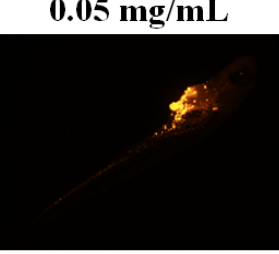

$5 \mathrm{mg} / \mathrm{mL}$
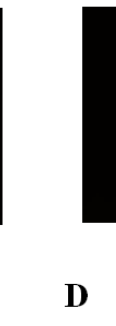

$0.5 \mathrm{mg} / \mathrm{mL}$

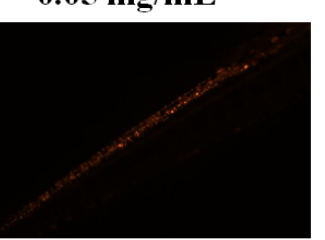

$5 \mathrm{mg} / \mathrm{mL}$

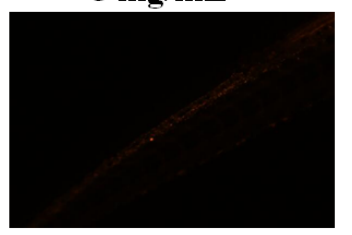


Figures

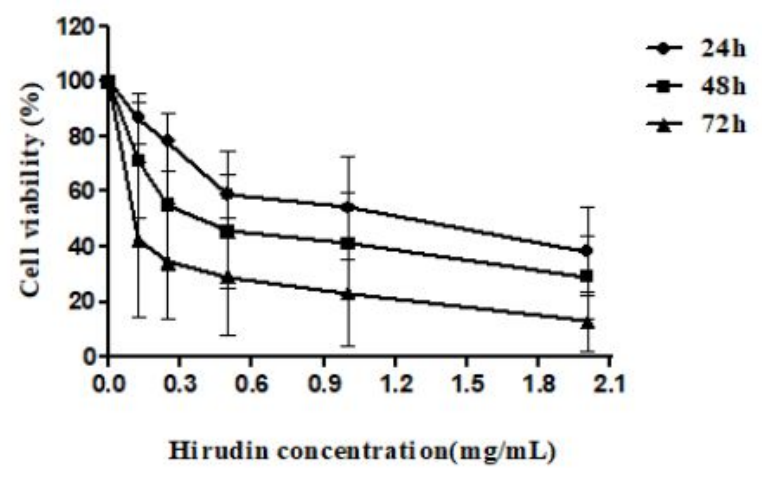

Figure 1. Hirudin induces growth inhibition of human breast carcinoma cell line MCF-7 following exposure at different concentrations at $24 \mathrm{~h}$, $48 \mathrm{~h}$, and $72 \mathrm{~h}$. Results are presented as the mean $\pm \mathrm{SD}$.

\section{Figure 1}

Hirudin induce s growth inhibition of human breast carcinoma cell line MCF 7 following exposure at different concentrations at $24 \mathrm{~h}, 48 \mathrm{~h}$, and $72 \mathrm{~h}$. Results are presented as the mean \pm SD. 


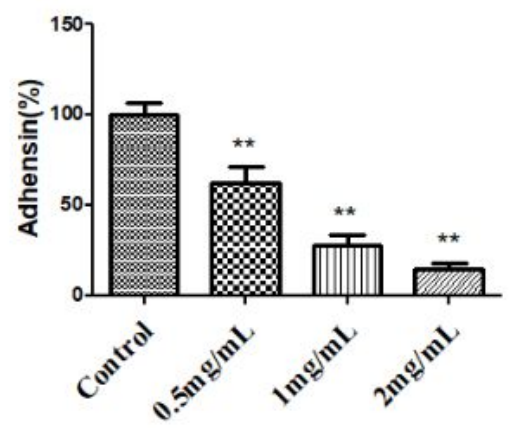

Figure 2. Hirudin inhibits the adhesive ability of MCF-7 cells. Data are expressed as a percentage in adhered cells treated with hirudin relative to the control cells. Results are presented as the mean \pm SD. ${ }^{* *} p<0.01$ vs. control group.

\section{Figure 2}

Hirudin inhibits the adhesive ability of MCF 7 cells . Data are expressed as a percentage in adhered cells treated with $\mathrm{h}$ irudin relative to the control cells. Results are presented as the mean $\pm S D$. $p<0.01 \mathrm{vs.}$ control group. 

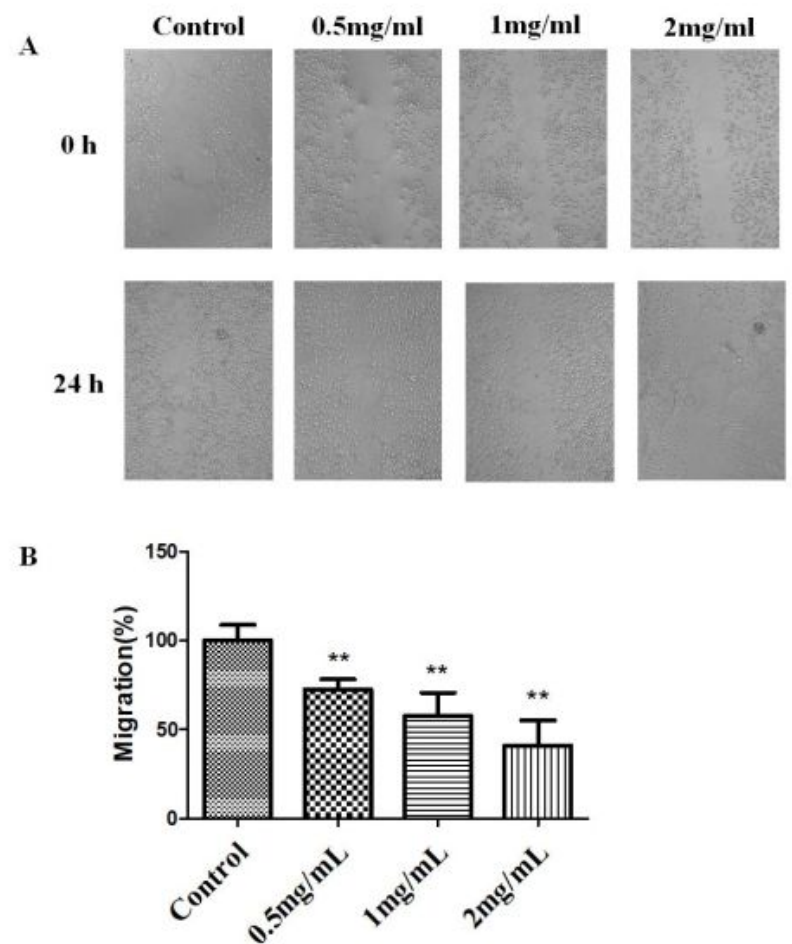

Figure 3. Hirudin inhibits the migration ability of MCF-7 cells. (A) Representative images of wound healing at 0 and $24 \mathrm{~h}$. (B) The percentage of migrated cells were calculated based on the number of untreated cells, which were set as $100 \%{ }^{* *} p<0.01$ vs. control group.

\section{Figure 3}

Hirudin inhibits the migration ability of MCF 7 cells. ( Representative images of wound healing at 0 and $24 \mathrm{~h}$. (B) T he percentage of migrated cells were calculated based on the number of untreated cells, which were set as $100 \%$. $p<0.01$ vs. control group. 

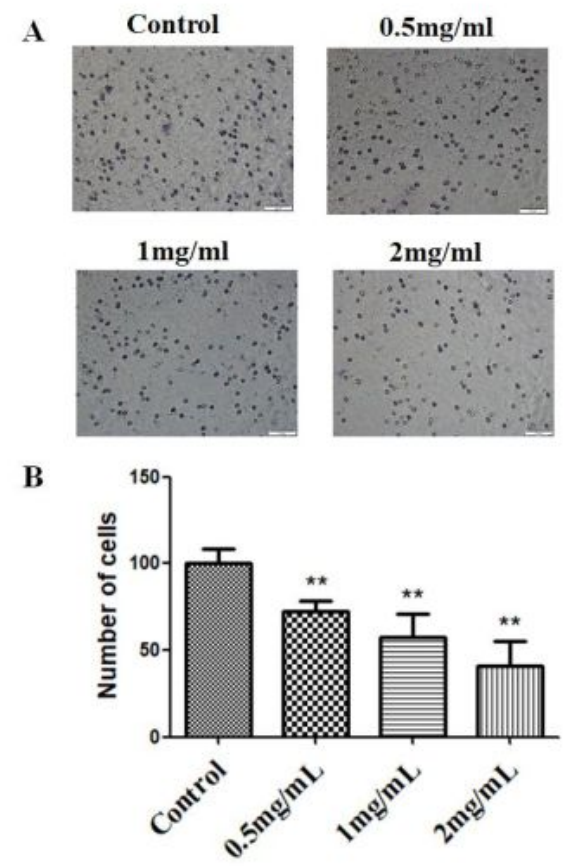

Figure 4. Hirudin inhibits the invasion ability of MCF-7 cells. Representative images (A) and data statistics of cells that invaded through the Matrigel coated Transwell chamber (B). ${ }^{*} p<<0.01$ vs. control group.

\section{Figure 4}

Hirudin inhibits the invasion ability of MCF 7 cells. Representative images (A) and data statistics of cells that invaded through the Matrigel coated Transwell chamber ( $p<0.01$ vs. control group. 


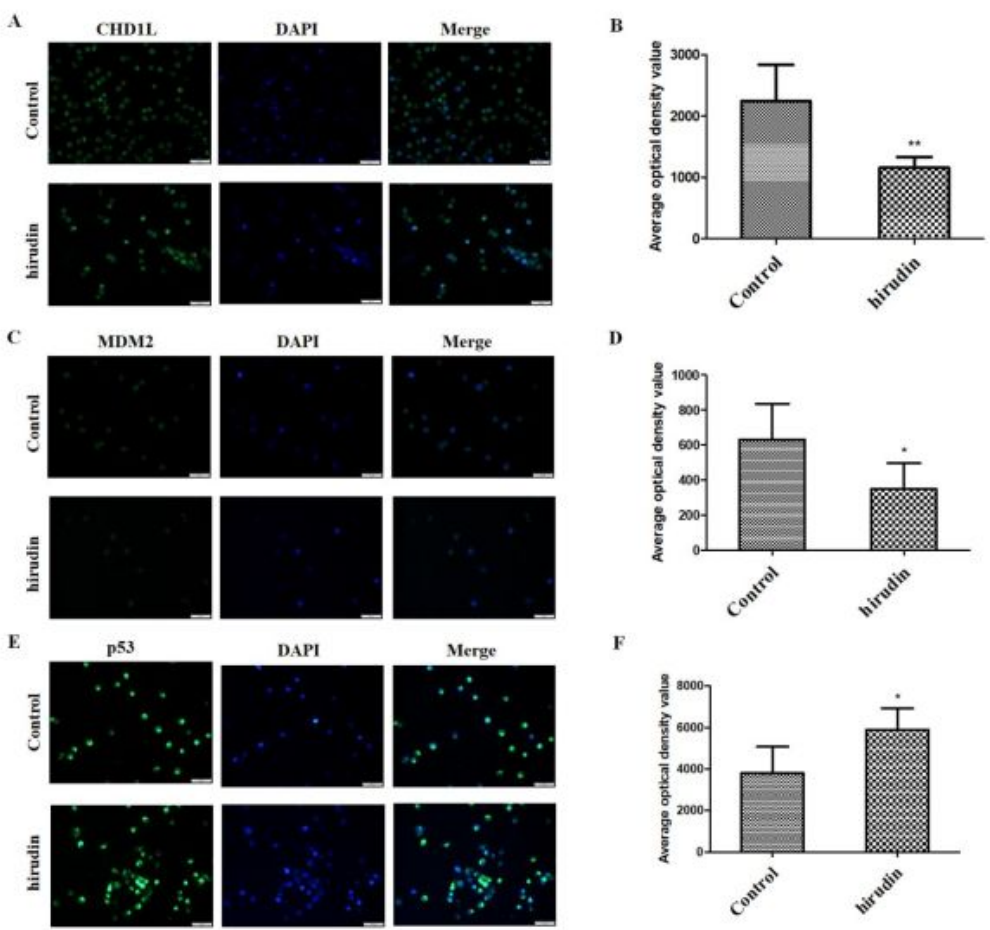

Figure 5. Hirudin suppressed the protein levels of CHD1L and MDM2, upregulated the protein levels of p53. Representative images (A, C, E) and data statistics of average optical density value (B, D, F). ${ }^{*} p<0.05$ vs. control group; $* *<<0.01$ vs. control group.

\section{Figure 5}

Hirudin suppressed the protein levels of CHD1L and MDM2, upregulated the protein levels of p53. Representative images $(A, C, E)$ and data statistics of average optical density value $(B, D, F) . p<0.05$ vs. control group; $p<0.01$ vs. control group. 


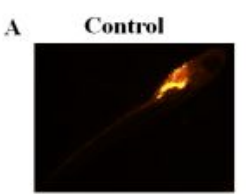

$0.5 \mathrm{mg} / \mathrm{mL}$

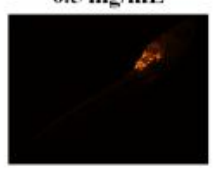

B

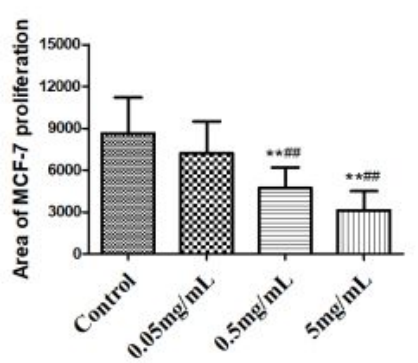

$0.05 \mathrm{mg} / \mathrm{mL}$

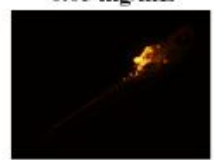

$5 \mathrm{mg} / \mathrm{mL}$

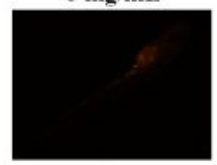

D

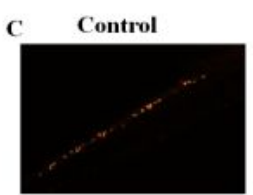

$0.5 \mathrm{mg} / \mathrm{mL}$
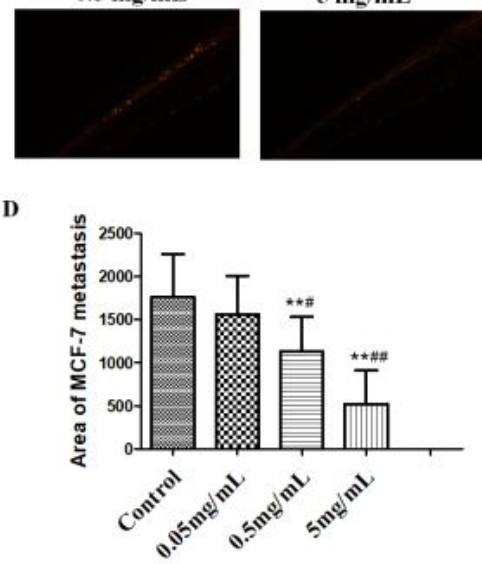

Figure 6. Evaluation of the in vivo antitumor effects of hirudin in zebrafish xenografted with the human breast carcinoma cell line MCF-7. (A) The in vivo growth is indicated by yellow stained tumor cells detected with the fluorescence microscope. (B) Area of MCF-7 proliferation. Results are presented as the mean $\pm \mathrm{SD}$. ${ }^{*} p<0.01$ vs. control group; ${ }^{\# \# p} p<0.01$ vs. $0.05 \mathrm{mg} / \mathrm{mL}$ group. (C) The in vivo metastasis is indicated by yellow stained tumor cells detected with the fluorescence microscope. (D) Area of MCF-7 metastasis. Results are presented as the mean \pm SD. ${ }^{* *} p<0.01$ vs. control group; ${ }^{*} p<0.05$ vs. $0.05 \mathrm{mg} / \mathrm{mL}$ group; ${ }^{\prime \prime} p<0.01$ vs. $0.05 \mathrm{mg} / \mathrm{mL}$ group.

\section{Figure 6}

Evaluation of the in vivo antitumor effects of hirudin in zebrafish xenografted with the human breast carcinoma cell line MCF 7 (A) T h e in vivo growth is indicated by yellow stained tumor cell s detected with the fluorescence microscope. (B) Area of MCF 7 proliferation. Results are presented as the mean \pm SD. $p<0.01$ vs. control group $p<0.01$ vs. $0.05 \mathrm{mg} / \mathrm{mL}$ group (C) T h e in vivo metastasis is indicated by yellow stained tumor cells detected with the fluorescence microscope. (D) Area of MCF 7 metastasis . Results 
are presented as the mean $\pm S D$. $p<0.01$ vs. control group $p<0.05$ vs. $0.05 \mathrm{mg} / \mathrm{mL}$ group; $p<0.01$ vs. $0.05 \mathrm{mg} / \mathrm{mL}$ group 